\title{
Reality in a few thermodynamic reference frames: Statistical thermodynamics from Boltzmann via Gibbs to Einstein
}

\author{
Vasil Penchev $^{1}$
}

\begin{abstract}
The success of a few theories in statistical thermodynamics can be correlated with their selectivity to reality. These are the theories of Boltzmann, Gibbs, end Einstein.

The starting point is Carnot's theory, which defines implicitly the general selection of reality relevant to thermodynamics. The three other theories share this selection, but specify it further in detail. Each of them separates a few main aspects within the scope of the implicit thermodynamic reality. Their success grounds on that selection. Those aspects can be represented by corresponding oppositions. These are: macroscopic - microscopic; elements states; relational - non-relational; and observable - theoretical. They can be interpreted as axes of independent qualities constituting a common qualitative reference frame shared by those theories. Each of them can be situated in this reference frame occupying a different place. This reference frame can be interpreted as an additional selection of reality within Carnot's initial selection describable as macroscopic and both observable and theoretical. The deduced reference frame refers implicitly to many scientific theories independent of their subject therefore defining a general and common space or subspace for scientific theories (not for all).

The immediate conclusion is: The examples of a few statistical thermodynamic theories demonstrate that the concept of "reality" is changed or generalized, or even exemplified (i.e. "degeneralized") from a theory to another.

Still a few more general suggestions referring the scientific realism debate can be added: One can admit that reality in scientific theories is some partially shared common qualitative space or subspace describable by relevant oppositions and rather independent of their subject quite different in general. Many or maybe all theories can be situated in that space of reality, which should develop adding new dimensions in it for still newer and newer theories. Its division of independent subspaces can represent the many-realities conception. The subject of a theory determines some relevant subspace of reality. This represents a selection within reality, relevant to the theory in question. The success of that theory correlates essentially with the selection within reality, relevant to its subject.
\end{abstract}

\section{INTRODUCTION}

A formal setting of how a scientific theory selects reality can be the following: Let $\mathrm{N}$ "things" share some M-dimensional space of their states so that $\mathrm{N}<\mathrm{M}$. Then $M-N$ dimensions and thus a corresponding $(M-N)$-dimensional subspace of the initial $\mathrm{M}$ dimensional space, in which all those "things" can be situated, are necessarily common. That subspace can be interpreted as the selection of reality relevant to the things in question and possibly

\footnotetext{
1 Dept. of Logical Systems and Models, Institute of Philosophy and Sociology at the Bulgarian Academy of Sciences, Sofia, Bulgaria; email: vasildinev@gmail.com.
}

studied by some scientific discipline and described by relevant and competitive theories.

The "things" can be interpreted as the elements of a set. The space of states is describable as a well-ordered set of "dimensions" (interpretable as qualities or quantities of the things). Any dimension has to have at least two discernibly distinguishable state being a quality and furthermore some oneto-one mapping into a number set being quantity rather than quality. The mathematical concept of vector space is an example of that kind of space.

In other words, the selection of reality relevant to some scientific theory or theories is seen as sharing a few common and inherent qualities or quantities of the things studied. Thus the selected reality corresponds to the extension of the theory, i.e. all common properties and relations shared by all studied things and preferably only by them.

Furthermore, reality can be thought as dividing the space of states into two parts or subspaces: constant and variable.

Husserl's phenomenology and especially "eidetic reduction" was what paid attention to that constant and invariant part of reality "bracketing" the other, variable part. However the analogy to Husserl's phenomenology should stop here for his "phenomenon" as the corresponding constant and invariant part of intention, still more both "phenomenological and transcendental reduction(s)", are not yet relevant to our discussion remaining in the scope of realism.

One can think of reality selected by some scientific theory also as a partial, but working concept of reality addressed just to that theory. If that is granted, its intension is the constant and invariant part of the space of states described by the theory, and its extension is the other, the variable part. Then the general sense of the concept of reality valid for any partial reality of any scientific theory is just that dividing the space of states into two disjunctive and exhausting subspaces corresponding to those constant and variable parts.

In other words, selecting relevant reality means the choice of a relevant boundary between the variable and constant, and thus between extension and intension to be defined rather implicitly by the context of the theory as a whole.

In fact, the practicality of selecting reality is only relative, in relation to another, parent or competitive, and thus commeasurable theory. That implies a comparison will select a few or even only one dimension(s), which change their (its) modus: from the constant part (intension of the partial reality) to the variable one (extension) or vice versa.

The core of that approach is to be substituted the general and in fact metaphysical problem about reality at all by the practically useful and in fact methodological issue about the change of the concept of partial reality between two (or more) very close and 
thereupon commeasurable theories. Consequently, selective realism is interpreted in thus in a fundamentally relative rather than absolute way: What are selected are only a few dimensions of the space of states changed its modus: either from intentional to extensional or vice versa.

That kind of selective realism is used as a methodology to be compared the implicit concept of partial reality in three successive theories in statistic thermodynamics: those of Boltzmann, Gibbs, and Einstein. All of them, being some additional specifications of thermodynamics, are naturally situated in the reference frame of Carnot's theory.

The implicit concept of the corresponding partial reality has been changed is changed as follows:

0. (Carnot) Classical thermodynamics describes laws in terms of quantities of that reality, which is as macroscopic as empirically and experimentally observable.

1. (Boltzmann) The mechanical motions of the huge number of microscopic elements of a statistical ensemble result into the thermodynamic quantities of any macroscopic physical object averagely. The empirically and experimentally observable quantities are deduced as derivative from a hidden theoretical reality of microscopic elements such as atoms and molecules.

2. (Gibbs) The mechanical motions of the huge number of microscopic elements are substituted by different possible states of a macroscopic physical object equivalently and mathematically. The empirically and experimentally observable thermodynamic quantities are deduced as derivative from a hidden theoretical reality of different possible macroscopic states of the physical object as a whole.

3. (Einstein) The mechanically and experimentally observable thermodynamic quantities are some function of the Gibbs ensemble of all possible states (and thus some relation to it). They can be furthermore also referred to the Boltzmann ensemble of microscopic elements. Reality includes both the observable object and the hidden theoretical model as whether a Gibbs or a Boltzmann ensemble as well as the function or relation between the object and that model.

The following conclusion can be deduced: Reality in those reference frames can be identified in the following oppositions: macroscopic - microscopic; elements - states; relational - nonrelational; observable - theoretical:

0. (Carnot): Macroscopic, both observable and theoretical.

1. (Boltzmann): Microscopic, elements, non-relational, theoretical.

2. (Gibbs): Macroscopic, states, non-relational, theoretical.

3. (Einstein): Both macroscopic and microscopic, both elements and states, relational, both observable and theoretical.

One can admit that still one synthesis has happen later to that reality, which can be utilized in a statistical and thermodynamic theory: both relational and non-relational. All other syntheses, which are implicit in the development of the concept of statistic and thermodynamic reality before it, are already completed in the Einstein theory.

One hypothesis might be that quantum statistical thermodynamics is what accomplished that last synthesis along that it involves still one dimension of another opposition as to reality: continuous (smooth) - discrete (quantum). All four theories mentioned above mean the thermodynamic and mechanical quantities implicitly only as continuous (smooth) though some of them introduce discrete elements.
One can summarizes the case study comparing the three theories in thus: The concept of "reality" is changed or generalized, or even exemplified (i.e. "de-generalized") from a theory to another. The change can be described as the explicit introduction of some new opposition as a still one and new dimension of relevant reality, and the generalization as a synthesis to some already involved opposition so that the theory is invariant to the relevant dimension of reality. The exemplification can also be observed being a condition for introducing a few new dimensions of reality. Thus, that exemplification simplifies reality in a dimension ("a step back") complicating it in a few others ("two steps forward").

The paper is organized as follows. Section 2 situates the utilized version of selective realism and specifies it as a methodology for the case at issue. Section 3 selects those dimensions of the partial reality in Carnot's theory, which will be modified by the three following theories. Sections 4,5 , and 6 try to define explicitly the implicit change of partial reality correspondingly in Boltzmann's, Gibbs's, and Einstein's theory. Section 7 discusses and tests the hypothesis that quantum statistical thermodynamics synthesizes the opposition "relational - non relational". Section 8 unites the changes of partial reality in each theory into a single viewpoint, presents the conclusions and provides directions for future work.

\section{DIFFERENTIAL REALISM IN THE REFERENCE FRAME OF SELECTIVE REALISM}

The term of selective realism is rather uncertain. For example, it is also utilized in theory of literature and art, meaning that some elements of the work are realistically represented unlike others in order to be achieved certain artistic suggestion. Their selection is a conscious and creative act of the author.

Its utilization even only in the framework of philosophy of science remains too wide, though. One can speak rather of family resemblance in its uses emphasizing common marks such as:

$\checkmark$ Scientific realism is the generic term.

$\checkmark$ One or more scientific domains, theories, parts of theories, and even only statements are or can be selected according some criteria as more or less realistic unlike others of the same kind: "Defenders of this form of realism typically separate theories into components or aspects according to some criterion such as structure or core descriptions or what have you and argue that only the selected components are eligible for realist claims, while components not thus selected (so-called 'idle' components) may be 'false' or 'non-referring', or simply 'idle' for whatever reason, without any serious implications for realism" [1].

$\checkmark$ Being "realistic" most often means to be represented rather successfully by certain relevant models in the item(s) at issue.

$\checkmark$ The term is a synonym of partial or limited realism. It emphasizes the realistic items in the background of the rest and can imply certain relations between them.

$\checkmark$ Its intention is to weaken the concept of scientific realism in accordance with the real history of science.

$\checkmark$ Key phrases for it might be: scientific realism, selection, criteria for selection, and relations of the selected and unselected.

"Selective realism comes in many variants: structural realism", "semi-realism", "divide-et-impera", "eclectic realism" [2]. 
Examples of heterogeneous uses in philosophy of science can illustrate the range of its possible implicit meaning:

Referring to the use of the same term of another author is often [3], [5], [6], [7], etc.

A "selection of scientific disciplines" relevant to scientific realism unlike others such as economics also can be meant: "scientific realism becomes the philosophy of the privileged class of successful (physical) sciences" [4].

One can discuss "the well-known causal properties of some entity" as independent of their interpretation and thus selectable realistically or not [5]. The selection of consistent objects in the background of inconsistent ones including in the context of mathematical realism is criticized as "dangerous" and "unstable" [6].

The term of "selective anti-realism" is also coined [7]. The "piecemeal realism" [8] is considered as a synonym of "selective realism" [7], and qualified namely as "selective anti-realism" emphasizing the non-realistic background of the selected as realistic.

P. Humphreys [9] offers "a form of selective realism", "which denies that one can simply read the ontological commitments from the theory itself". Its user is who interprets it realistically, but utilizes always only a part of it. The use itself of the theory selects used and idle parts: "only idle parts of past theories have been rejected, while truly success-generating features have been confirmed by further inquiry" [10]

"Selective realism seems to make good sense as a "working hypothesis', but as no more than that." [11] The theories are successful in some sense. Their success can be causally explained reconstructing selectively those elements caused it. "So-called 'selective' realists attempt to explain various successes in the history of science by reconstructing the relevant derivations so as to show that the success of a given theory depends only on those things it actually got right (be those things properties, structure, or whatever)." [11] "Success is supposed to provide a reason for believing that particular aspects of a theory are at least approximately true. Selective realism thus requires not just continuity, but a particular type of continuity: the retention of those parts that are differentially supported by the successes of our theories." [12] Success should be investigated comparatively. "Perhaps if we understand scientific success as a comparative achievement, we will find that success tracks the stable elements of our evolving theories more reliably than if we interpret success non-comparatively." [13]

"Selective realists think that there is not a distinct universal for every predicate. How then do they choose which predicates have distinct universals?" [14] That criticism discusses selective realism in logical, onto-logical and linguistic terms doubting the justifiability of the criteria of selection. "Whereas an unselective realist is committed, roughly, to there being a universal for every predicate, a selective realist is committed to there being one for some but not all predicates." [15]

Selective realism correlates with "retail realism" and "retail treatment of theoretical entities" [16] In short, then, wholesale treatments lead to either wholesale acceptance or wholesale rejection of a theoretical entity as it appears in a number of distinct theories, while retail treatments allow for the possibility of proceeding on a case-by-case basis, which can lead to a kind of selective realism about some theoretical entities, but not others." [16]
One of the most attackable elements of any version of selective realism is the criteria for selection. D. Peters argues that "the essential posits of a theory are those that unify the accurate empirical claims of that theory" [17] There is "the basic rejection by selective realism of the view that theories should be treated as holistic units for the purpose of realist commitment" [17]. Is the selection of parts of any scientific entity at all admissible?

Some authors discuss aspects of selective realism under other names [18], [19] or implicitly, in the context of other discussion [20].

An article [21] discusses especially selective realism in physics and its philosophy: "selective realism rests on a causal test whose application is itself part of the scientific enterprise" [21]. However the success of a scientific theory goes out of the "scientific enterprise" being a rather social and psychological phenomenon. "Selective realism is realistic realism, that is realism more true to scientific life than any blanket, undiscriminating doctrine." [21] Whether scientific life is more selective and discriminating e.g. than life in economy or in politics? "One main aim of any selective realism must be to find a principled way of distinguishing, among items figuring in scientific theories, those which must, and those which need not, or even must not, be given a realistic interpretation. This search for a criterion for the real must be understood as a search for a criterion for us to count something as real ..." [21] In fact being real is always a relation between models only normatively independent of each other, e.g. an interpretation of experimental data and a forecast of a theory.

One can reflect on the above bricolage of uses of "selective realism". First, it is a selection of sources accessible as to me. Secondly, it is still one following selection of uses most relevant to mine. Thirdly, it can be considered as a proto-concept or germ of theory about selective realism.

This hints that any realism is selective, but does reflect on its hidden selection far not always. Indeed all authors of scientific papers, some of which will be legitimated as theories, select what is the most important among the accessible and might guide to the cherished success of legitimation observing and comparatively estimating the competitors.

Furthermore, all above uses though selected are only on the road to a single concept or theory. They share only some family resemblance. Achieving the endpoint of a single and consistent theoretical entity is quite not the most optimal strategy for success.

The author should select the best point for the "camp" of the intended paper on that road from the absolute uncertainty to the absolute certainty: the more uncertain, the more universal, flexible, and adaptable; the more certain, the more stable, forecasting, and efficacious.

The normatively welcome consistency meets the inconsistent circumstances of the real science. The latter is even more important for the paper to survive. It should sneak out safe and sound among both dense and inconsistent networks of reviewers, funding institutions, scientific authorities, influences, "paradigms", impact factors, criticisms, etc.

Consequently, any reasonable authors select some optimal uncertainty for the representation of the results over the unintended inconsistency due to their foolishness.

However and post factum, philosophy and history of science are interested in the consistent and "approximately true" parts of the theories, i.e. of the most successful papers, selecting only them as relevant to realism. One can suggest the hypothesis that the 
only consistent and thus realistic part of a paper might never sneak out standalone through the real science, though:

Selective realism selecting only consistent and approximately true parts is not realistic. Any paper achieves success as a whole and even as a member of the team of similar papers of the same and other authors, and the alleged inconsistent or idle parts or papers could be exceptionally useful, working and adequate to certain real circumstances.

However and post factum, the philosophers and historians of science might hardly reconstruct those real circumstances, to which some inconsistent and idle parts or papers could be too adequate and thus promoting the whole. Anyway what can the realists do really without transforming into social constructivists or agnostics?

The approach in this paper is the following: Relative and temporally close theories or hypotheses to be investigated comparatively hoping that they had shared common though unknown real circumstances. If one takes their difference or change from each other, those unknown real circumstances might cancel mutually. This implies that the only, which makes sense to be selected, is the difference and change of maximal relative and temporally close theories and hypotheses.

One can coin correspondingly the term "differential realism" as to that version of selective realism.

Furthermore, the entire team (consisting whether of scientists or of different papers of one and the same author(s), or of parts of any scientific entity) seeker for scientific success is better to participate in the competition with a "combination" including both a constant core and a variable belt of hypotheses. Though only a few and even only one member(s) of the combination can win, the team cannot know preliminarily which exactly, and the combination increases the probability of luck.

However, the reconstructing selective realist would be to qualify the luckless members of the combination as inconsistent or idle and thus as useless, which is obviously incorrect.

What should a differential realist do in that case?

Any scientific theory or hypothesis can be thought as a single notion defined in a rather extended way, and the definition of which is merely very, very long. Of course, its length increases the probability of being inconsistent. Let us admit that its definition is nevertheless consistent for even being inconsistent, it can be divided into disjunctive consistent parts and each of them can be considered separately.

Then any notion can be interpreted as a certain relation of its extension and intension. The definition represents its intension and should unambiguously determine its extension. Furthermore, the intension and extension can be united into a common space as its disjunctive subspaces. The components for the intension are constants, each for each dimension of the corresponding subspace, and the components for the extension are variables, each for each dimension of the corresponding subspace. Thus the different members of the combination will differ from each other by a few dimensions passed from the extension to the intension subspace or vice versa.

The differential realist should discuss those a few problematic dimensions unlike the reconstructing selective realist paying attention only to the member winner. In fact, the attitude of the differential realist is much closer to that of the real scientist(s) creating the combination seeker for scientific success.

Furthermore, wrong principles and hypotheses can influence the development of science much more than correct theories. For example, Einstein's "Mach's principle" in general relativity or alleged incompleteness of quantum mechanics (EPR) seem to be both wrong and exceptionally fruitful, stimulating research generated new scientific ideas and areas: the cosmological constant, the hidden "dark energy" and "dark matter", and entanglement studied by the theory of quantum information nowadays. However as being wrong, they cannot seek for scientific success, according to the reconstructing selective realist.

On the contrary, this is not any problem for the differential realist. The explanation of the indirect success of such wrong hypotheses is quite natural and simple: They pay attention to a few most essential dimensions of reality though they have not managed to target the lucky combination. Others will manage ...

Scientific realism is in interested in reality at all, selective realism in the selected part of reality, differential realism in the small changes of the implicit concept of reality from a theory or hypothesis to another very close and relative.

\section{THE PARTIAL REALITY OF CARNOT'S THEORY AS A REFERENCE FRAME OF CHANGE}

Carnot's theory of heat should be investigated comparatively in relation to its predecessors or contemporaries: the theories or hypotheses of B. Thompson (Count Rumford), Davy, T. Young, J. Herapath, A. Lavoisier, S.-D. Poisson, J. B. J. Fourier, etc.: "The dominating theory of the time was the caloric theory. Whereas in England caloric theory met its opposition by the semimechanical theories of Davy, Young, and Herapath, exerting their authority by invoking Newton, in France there was an almost complete acceptance of caloric theory, based on the authoritative personalities of the likes of Lavoisier, Poisson, and Fourier." [22] R. Fox drew attention to "Watt's expansive principle in the Work of Sadi Carnot and Nicolas Clement" (together with Charles Bernard Desormes) [23].

However, those theories in turn should be discussed in the reference frames of their predecessors, and so on to a kind of "bad infinity". This imposes the beginning to be defined axiomatically, i.e. by a few most important properties or relations granted for postulates.

One can criticize that approach as teleological and constituting a vicious circle: the axiomatic properties or relations are selected, meaning its application to the theories of Boltzmann, Gibbs, and Einstein, the implicit concepts of reality in which are to be deduced by those axioms. The objection cannot be accepted for it is valid to any application of deductive and axiomatic method, one of the "three whales" of mathematics.

The selected postulates about the implicit concept of reality in Carnot's theory are the following:

1. Reality is both empirically (and experimentally) observable and theoretically describable by quantitative models.

2. Reality is given immediately in macroscopic phenomena.

In fact, both postulates had been shared by all physical theories from the age of Galileo and Newton. So, they do not need any express ground. Boltzmann was who revolutionized that implicit concept of reality paying the worth of his tragic death.

The core of Carnot's work and contribution is the cycle called it his honor, the concept of heat (both "le calorique" and "la chaleur"), the unity of mechanical work and heat, the postulated 
impossibility of perpetuum mobile, the first ${ }^{2}$ and second laws ${ }^{3}$ of thermodynamics, etc.

However any "translation" (as above) of his work [24] into terms and notions of the contemporary "phenomenological thermodynamics" is too dangerous and should serve only for a preliminary orientation.

A relatively successful attempt of that kind concludes: "CARNOT laws: I. Perpetuum mobile is impossible; II. Heat is conserved". "Thermodynamic laws: I. Energy principle [conservation]; II. Perpetuum mobile of second kind is impossible". And then: "While irreversible processes in thermodynamics are described by the second law, they are in CARNOT'S theory described by the first law. It follows especially that the two theories are equivalent as long as only reversible processes are considered ..." [25]

G. Sarton [26] drew attention to an unpublished manuscripts of Carnot lucidly revealing that Carnot had anticipated not only energy conservation (the first law of thermodynamics), but also the main idea of the Boltzmann statistic thermodynamics:

La chaleur n'est autre chose que la puissance motrice, ou plutot que le mouvement qui a change de forme. C'est un mouve- ment dans les particules des corps. Partout oiu il y a destruction de puissance motrice, il y a, en meme temps, production de chaleur en quantite precisement proportionnelle 'a la quantite de puissance motrice detruite. Reciproquement, partout oiu il y a destruction de chaleur, il y a production de puissance motrice.

M. Barnet tried to explain Carnot's fundamental innovating as to the nowadays Second Law of thermodynamics by the auxiliary notions of available and unavailable type of energy [27]: The former was referred to the parts of a system, and the latter to the system as whole. For example, kinetic mechanical energy should be of the former type, and potential one of the second. Energy conservation and the First Law postulate their equivalence and reversibility. On the contrary, the Second law states their irreversibility: "available" forms of energy are capable of complete transformation into those of the "unavailable" type, the extent of transformation of the latter into the former can never, even under ideal (reversible) conditions, exceed a definite maximum which must always fall short of complete convertibility" [27]

Thus the Second Law would contradict to the First Law if the available and unavailable types of energy are not divided into two disjunctive physical quantities such as energy (or work, or heat, or "la chaleur") and entropy (or "le calorique") therefore implying the quantity of their relation (or ratio): temperature. "Sadi Carnot had, however, formulated the basic ideas relevant to the second law of thermodynamics at a time when the caloric theory of heat was reigning supreme" [28]

Another attempt for that translation of Carnot's theory into the language of thermodynamics finds "correspondence between entropy and caloric [calorique]" that "may serve as a very effective heuristic tool for finding the properties of caloric by

\footnotetext{
2 "However, SADI CARNOT (1796-1832) was not simply the discoverer of the second law; he had also anticipated with astounding directness and lucidity the discovery of the first law." [26]

3 "We have identified in Carnot's essay a principle that, with conservation of caloric, played the role of the second law of thermodynamics. This principle states that the simplest possible cyclic heat engine is one that
}

exploitation the results known hitherto from classical thermodynamics" [29]. The physical quantity of caloric [calorique] should represent the mechanical work per degree fall in a Carnot cycle, according to H. L. Callendar [30], and thus the physical dimension of caloric would be that entropy, too:

"Carnot's cycle thus represents the conversion of thermal energy (TS) into mechanical work A by the fall of entropy through a potential difference $\left(T_{2}-T_{1}\right)$

\section{$\partial A_{\text {thermal }}=\partial S\left(T_{2}-T_{1}\right) "[31]$}

J.N. Brønsted [31] shared the same interpretation of caloric as entropy and developed a common theory about the reversible cycles of extensive quantities ${ }^{4}$ such as entropy, mass, electric charge through the conjugate potential difference are necessary for the production of work. Thus Carnot's cycle was rather generalized from "caloric" to all extensive physical quantities.

One of the main problems of that interpretation is how "perpetuum mobile" in his work should be interpreted in relation of the contemporary distinction between that of first and second kind; another: what should be relation between the two terms of "le calorique" and "la chaleur", and the only contemporary term of "heat" (or "heat energy"). In particular, this demonstrates that the unambiguous, one-to-one translation is impossible.

Fortunately, one can obtain that unambiguousness considering "heat" and "perpetuum mobile" only as the differential dimensions of reality in the difference or change between the two theories: The "heat" dimension is in the intension of the contemporary phenomenological thermodynamics, but in the extension of Carnot's theory having vales as "le calorique" as "la chaleur".

On the contrary: the "perpetuum mobile" dimension is in the extension of the contemporary phenomenological thermodynamics having values as "first kind" as "second kind", but in the intension of Carnot's theory.

Thus the comparison between these two theories naturally selects the dimensions both of "perpetuum mobile" and of "heat":

If one needs a one-to-one translation for some reason, both "first and second kind", on the one hand, and "le calorique" and "la chaleur", on the other hand, should be reciprocally identified.

If one needs the differential research of the two theories, the relations in this two-dimensional subspace should be investigated in detail. That differential research might prove or refused any more extended and even complete equivalence of phenomenological thermodynamics and Carnot's theory than the common one about reversible processes:

"A deep symmetry exists between the full system of equation of modern thermodynamics and those of the caloric theory - not that anybody wrote them down, but the flexibility and the near correctness of that theory are manifest." [35]

Indeed the relation of heat (chaleur) and caloric (calorique) in Carnot's theory, on the one hand, and that of perpetuum mobile of first and second kind in phenomenological thermodynamics, on the other hand, can be both interpreted as temperature and then entropy conservation per a unit of time to be equivalent to energy

produces work by extracting heat from one heat reservoir and rejecting heat to a cooler heat reservoir." [33]

${ }^{4}$ The extensive quantities are proportional to the quantity of parts of the system, and the intensive ones (such as the corresponding conjugate differences of temperature, gravitational or electrical potential) to its whole. 
conservation by the meditation of the Planck constant and quantum mechanics [36].

Even more, Carnot's theory of heat would turn out to be the more general one for it implies a generalization of energy conservation to that of calorique, i.e. of entropy (information): energy conservation would be equivalent to the linear increasing of entropy in time.

The main problem after reconstructing his work is: "How did Carnot know how to close his cycle?" [22] Indeed all great contributions to thermodynamics ${ }^{5}$ and its foundation enumerated above are immediate corollaries ones the cycle has been closed.

The fact of closing the cycle had been obvious to Carnot and even to his contemporaries for it is implied by the cycle of steam engine, which is obviously closed, and the investigation of which is the problem starting point for Carnot:

In order to consider in the most general way the principle of the production of motion by heat, it must be considered independently of any mechanism or any particular agent. It is necessary to establish principles applicable not only to steamengines but to all imaginable heat-engines, whatever the working substance and whatever the method by which it is operated. [24]

According to C. Truesdell and S. Bharatha, thermodynamics can be based on certain axioms in a way that "the theory of heat engines is not an application of general principles but the source of them. The traditional "First Law" and "Second Law" of thermodynamics, for fluid bodies susceptible only of "reversible" processes, appear here as proved corollaries of theorems on heat engines..." [37]

Consequently, the extended "notion" of Carnot's theory represents the consistent theoretical description generalizing the work of steam engine. This is the change to predecessors' and contemporaries' hypotheses about heat.

However this change is granted and shared by Boltzmann's, Gibbs's, and Einstein's theories and thus is irrelevant to their differential research here though it would be absolutely relevant if the topic was "Carnot's revolution in (of) thermodynamics (heat theory)".

These three theories will separate and then will seek for ways the observed macroscopic thermodynamic phenomena and the hidden theoretical reality to be again united as they were in the innocent paradise of Carnot's theory. This is what determines the selection of the above two postulates about the implicit concept of reality in Carnot's theory.

Nonetheless, the above two postulates about the implicit conception of reality in Carnot's theory imply its achievement: the equivalence of the cycle of steam engine and the theoretical Carnot cycle and thus the foundation of thermodynamics:

Indeed the steam engine and its cycle can be relevant to the partial reality of Carnot's theory (according to 2), and they can be identified with Carnot's cycle (according to 1). Then Carnot's cycle should be closed just as its material proto-image, the cycle of steam engine really is, and after that and all so important corollaries deduced.

\footnotetext{
5 "It was found that in a number of extremely critical positions, thermodynamics is not at all considered an indisputable continuation of Carnot's ideas. Moreover, present difficulties in understanding such basic
}

However, this implicit concept of reality is not contributed by Carnot, but borrowed from the other physical theories then. The success of Carnot is its application in a new area therefore constituting a new scientific discipline, thermodynamics.

The reconstructing post factum scientists will wonder "How did Carnot know how to close his cycle?" [22]. However their problem could be his for his implicit concept of reality could not include the posterior separation of the macroscopic phenomenological reality from the theoretical one in thermodynamics due to the theories of Boltzmann, Gibbs, and Einstein, which imply that problem.

These theories are certainly well-known to the reconstructing nowadays. So their reconstruction turns out to be from the future of Carnot's theory and thus only pretending to seek for a causal explanation of success.

This is a fundamental and inherent problem of the reconstructing selective realism: How might it prove that its reconstruction is not influenced by theories, facts and events future to the reconstructed entity. I think hardly...

\section{BOTLZMANN'S THEORY AND ITS CHANGE IN THE IMPLICIT CONCEPT OF REALITY}

Boltzmann postulated a hidden reality consisting of invisible mechanical particles, atoms and molecules, however determining our empirical and experimental reality of phenomena in thermodynamics:

The idea of some hidden and determining reality had been quite familiar in European philosophy at least since the age of Plato and Democritus, and even earlier.

"Boltzmann's philosophy was realism, or, as at the end he occasionally called it, materialism. He derived his conviction of the reality of the external world as an object of science from what may be called "concordance". The information obtained by different senses, with the help of different instruments, by different persons, and at different times, always lead to similar conclusions about the external, real, world. This could not happen if the independent world had no objective existence." [38] "Boltzmann emphasizes that his views are in harmony with those who argue for the existence of God" [39]

Atoms and molecules had been established enough notions in chemistry since John Dalton and especially after Mendeleev. They were unobservable and thus remained purely theoretical constructs, but their supposed reality could explain exceptionally simply and entirely the properties of the chemical elements, compounds and possible reactions, and even forecast successfully new ones thus converting chemistry into an exact science as physics.

However physics had managed to become a deterministic science of precise predictions and engineering applications for a long time utilizing mathematical model immediately to macroscopic phenomena and thus not needing atoms and molecules so greatly as chemistry.

In particular, phenomenological thermodynamics had been borrowed and wealthy repeated the general philosophical approach to reality of other physical disciplines. Carnot and Clausius and many others even before them had already deduced

thermodynamic parameters as heat energy, heat, work, and, especially, entropy are caused to a large degree precisely by Clausius' rejection of some of Carnot's fundamental ideas." [33] 
mathematical laws and relevant quantities about empirical and visible thermodynamic phenomena.

So, the statistical methods to thermodynamics, which Clausius, Maxwell, Helmholtz ${ }^{6}$, and Boltzmann addressed, seemed to be both risky and not enough justified. Nonetheless, these ideas were the first steps on the road, which would opposite physics to common sense, most obviously in quantum mechanics.

That certain hidden theoretical reality absolutely different from ours, empirical reality and nevertheless grounding it by fantastically precise forecasts and technical applications was that dangerous philosophical invention, in which Boltzmann would situate [40] his new theory.

The corresponding implicit concept of reality can be placed in the reference frame of Carnot's as follows:

They are opposed in size: Carnot's is macroscopic, visible and Boltzmann's is microscopic, invisible even by microscope (in that time). Until, then the size is not a dimension of reality: Both big and small items shared common reality.

Consequently, Carnot's is observable, and Boltzmann's is not: Anyway, the latter is also accessible, but only by theoretical, especially mathematical models.

These models in Boltzmann's theory are testable and thus verifiable by their corollaries producing observable forecasts in our usual, empirical reality. "Boltzmann's Bildtheorie, which asserts that scientific theories are 'mental pictures' having at best a partial similarity to reality, was a core element of his philosophy of science." [41]

Thus the relation of model and reality abandons the naïve selfunderstandable one-to-one mapping between them. EPR's concept "element of reality", in order to be proved the alleged incompleteness of quantum mechanics, would restore the lost paradise of that kind of models.

On the contrary, the models in Carnot's theory are directly testable and verifiable by virtue of that one-to-one mapping.

Carnot's reality is holistic as a cycle is a whole. The concept of cycle, though borrowed from the empirical cycle of steam engine, helped for him to formulated those of caloric and the impossibility of perpetuum mobile referable to the whole of a thermodynamic system.

On the contrary, Boltzmann's reality consists of a simple sum of exceptionally many tiny parts: the "atoms" [39].

His entropy is restored by the distribution of the "atoms" (they can be any many tiny particles, e.g. molecules) in energetic states: Entropy (S) is proportional to the logarithm of the number (W) of microstates per a macroscopic state ("Boltzmann's principle"7). "The central quantity $\mathrm{W}$ is named thermodynamic probability. Boltzmann worked out the [above] prescription how to combine it with atomistics." [43]

\footnotetext{
6 "In a couple of papers [1. Helmholtz, H.: Principien der Statik monocyklischer Systeme. Crelle's J. 97, 111-140 (1884); reprinted in:Wissenschaftliche Abhandlungen, vol. III, pp. 142-162 and pp. 179202, Johann Ambrosius Barth, Leipzig (1895); 2. Helmholtz, H.: Studien zur Statik monocyklischer Systeme, Akademie der Wissenschaften zu Berlin, S. 159-177 (1884); reprinted in:Wissenschaftliche Abhandlungen, vol. III, pp. 163-172 and pp. 173-178, Johann Ambrosius Barth, Leipzig (1895)] on the mechanical foundations of thermodynamics, Helmholtz introduced a class of mechanical systems that was to provide a mechanical analogy to the second law of thermodynamics. Helmholtz's results inspired the contemporary Boltzmann paper [3. Boltzmann, L.: U" ber die Eigenschaften monozyklischer und anderer damit verwandter Systeme.
}

"It was this statistical interpretation of a basic law of nature which shocked many physicists ... " [39] However, the mechanical motion is reversible unlike the irreversibility of the second law of thermodynamics and seems to be unreliable as a universal ground of entropy increasing though only statistically. Boltzmann managed to find a simple, smart and convincing explanation [44]:

Any collision of atoms averages their mechanical quantities and thus "flattens" the function of their probability distribution. As the number of those collisions increases monotonically in time, the probability distribution will flatten and flatten also monotonically in time, and entropy will increase and increase accordingly. "In other words: any non-uniform distribution evolves into an almost uniform one after a long time $\boldsymbol{t}_{\mathbf{1}}$." [44]

Indeed the laws of mechanical motion are smooth and thus reversible, but not those of collision. Collison is jump-like. Many years later, quantum mechanics will manage to prove that the uniform description of smooth and quantum (i.e. jump-like) motion or change implies irreversibility.

And vice versa: the combined reversibility and irreversibility of the first and second laws of thermodynamics implies for the corresponding phase space to be "checked", the Boltzmann and Gibbs entropy not to be equal, thus thermodynamics of the whole and that of the parts to be emancipated to each other and therefore independent addressing the Einstein statistical thermodynamics [45].

"It should be remarked that earlier (in 1868) ${ }^{8}$ Boltzmann also mentioned Einstein's idea of basing the probabilities W of a system in equilibrium on the dynamics of the system, by postulating that the probability to find the system in a certain region in phase space would be proportional to the time spent by the system in that region, which clearly depends on the equations of motion. However, he never distinguished this from his statistical idea, based on $\mathrm{S}=\mathrm{k} \log \mathrm{W}$ of 1877 , as Einstein did." [45]

Boltzmann's "heat" is the average energy of the statistical ensemble. Pressure and volume are deduced also from the average mechanical quantities.

Heat, temperature, pressure, volume are only a macroscopic visibility or even "maya". There is nothing else than moving atoms, however still unobservable...: a too dangerous combination of radical, hardly acceptable philosophical views and missing facts and experiments about the "atoms" as to a scientific theory. Thermodynamics is statistical mechanics after Boltzman.

"It has been suggested that Boltzmann himself, as a result of the criticisms of his theory, abandoned his mechanistic philosophy and adopted a more pragmatic approach in which the real existence of entities like atoms was no longer important. The result of this revisionist view is to see Boltzmann as a convert to

Crelle's J. 98, 68-94 (1884); reprinted in: Hasen"ohrl, F. (ed): Wissenschaftliche Abhandlungen von Ludwig Boltzmann. Band III, $p$. 122 (1909); reprinted by Chelsea Publishing NewYork (1968)] in which the notions of temperature and pressure were introduced for a onedimensional mechanical system with periodic orbits and potential energy $\phi(\mathrm{x}, \mathrm{V})$ depending on a parameter V.” [42]

7 "This formula was called Boltzmann's principle by Albert Einstein. It is engraved on Boltzmann's tombstone in the central cemetery of Vienna ..." [41]

8 "L Boltzmann, Studien über des Gleichgewicht der lebendigen Kraft zwischen bewegten materiellen Punkten, Wien. Ber. 58, 517-560 (1868)." 
Mach's philosophy and to make him an honorary member of the Vienna Circle. Perhaps the best justification for this interpretation is Boltzmann's view that scientific concepts such as ,atom" are mental pictures rather than entities independently existing in the world." [46]

Boltzmann's reality is not holistic: it is even anti-holistic, reductionist. The relation between the whole and the parts is directly reduced to properties of the parts. The concept of the whole is even redundant, prescientific.

On the contrary, the idea of cycle in Carnot's theory harmonizes the whole and the parts in a natural, and maybe naïve way, so that one cannot ask about their relation.

Summarizing, the differential investigation of the implicit concept of reality in both theories needs the following dimension:

$\checkmark$ Size: microscopic-macroscopic.

$\checkmark$ The relation of the theoretical and empirical (experimental): opposition - coincidence

$\checkmark$ The relation of the whole and the parts: harmonized (cyclical) - non-relational reductionism (to the "atoms")

$\checkmark$ The relation of the models to reality: directly testable and verifiable - indirectly testable and verifiable (by corollaries).

In fact, some of marks as to Carnot's reality could be outlined after comparing with Boltzmann's. They have been available also before it, but the juxtaposition activates them by the necessity of selection and choice between just these two worlds among the many worlds of different theories.

One can say that the main philosophical problem of Boltzmann's theory is how the whole in phenomenological thermodynamics to be entirely represented in terms of a huge set of parts, "atoms" [46], thus reduced to them thoroughly and even de facto removed.

"As a physicist working mainly theoretically Boltzmann strove for a close relationship to philosophy und recommended such $u$ procedure also to his colleagues." [47] "Mind and matter are not separated; they are only different sides or faces of reality. We can distinguish but we cannot split them. There are no [purely] mental sciences [Geisteswissenschaften]. And it is wrong to think that the natural sciences lack direction in terms of mind or spirit." [48] His implicit concept of reality contains the solving of the problem, "Boltzmann's principle", being supported by European philosophy non- and even anti-holistic in comparison to the Chinese for example:

Indeed it guarantees an enough refuge (microscopic and invisible, theoretical and only mathematically accessible, indirectly testable and verifiable) for the unobservable atoms both to exist out of empirical reality and to determine and control it nevertheless.

\section{GIBBS'S THEORY AND ITS CHANGE IN THE IMPLICIT CONCEPT OF REALITY}

Gibbs came to the statistical ideas after many years of investigation in phenomenological thermodynamics following Maxwell's definition of it expressly "without any hypothesis as to the molecular constitution of bodies", to which paid attention M. Klein [49].

The metaphor that Gibbs's theory is Boltzmann's without atoms is a relevant starting point to it. Indeed the atoms in

\footnotetext{
${ }^{9}$ The concept of phase space in thermodynamics had been introduced yet by Maxwell [49], [50]. Consequently, the philosophizing physicist
}

Boltzmann's theory possessed many redundant properties idle to their role in statistical thermodynamics besides being undiscoverable. Boltzmann's principle in fact determines altogether their properties really necessary for statistical thermodynamics:

$\checkmark$ Their states called microstates are the measure units for the empirical observable macroscopic states to be compared to each other quantitatively in entropy.

$\checkmark$ Both macro-and microstates share the physical dimension of action, the time derivative of which is energy.

These two properties absolutely exhaust what statistic thermodynamics needs from the concept of atom. So, "phase space" $"$ allows of comparing thermodynamic macroscopic states or system getting rid of atoms' brokering.

Even much more, that new refined formulation of statistic thermodynamics turns out to be rather more general.

However Gibbs "did not invent the phrase "phase space" [51] Moreover, he did not use at all. His fundamental work [52] in statistical thermodynamics does contain it no one time. Then is not the attribution of "phase space" to his theory absolutely nonsense? Quite not, because he used a few other terms even stronger than the contemporary use of "phase space": "extensionin-phase" (a differential sell in phase space); "density-in-space" (the change of the number of systems or states sharing some extension(s)-in-phase); "the principle of conservation of extension of phase" in two forms: differential called "conservation of density-in-phase", and integral called properly "conservation of extension-in-phase" [52]. Indeed:

$\checkmark$ Introducing "extension-in-phase", he introduced implicitly phase space as the set of all extensions-in-phase. So he stressed that they are his elements, the counterparts of Boltzmann's "atoms" or "molecules".

$\checkmark$ Involving "density-in-phase", he demonstrated the way for the systems or states to be "built" by the "extensions-inphase", namely sharing them. The constant number of density-in-phase corresponds to the constant number of microstates per a macroscopic state in Boltzmann's theory.

$\checkmark$ The principle as conservation of density-in-phase states that density-in-phase is constant in time (as to an ensemble of identical mechanical systems under the action of identical forces). This means that the probability for a system to share some volume in phase space is constant in time and thus corresponds to thermodynamic probability.

$\checkmark$ The conservation of extension-in-time means action conservation rather than energy conservation. Indeed energy conservation cannot describe the energy exchange between extensions-in-phase and the system as a whole for the huge difference in their energies. On the contrary, the conservation of quantity of action ("phase") makes sense for the tiny energy of an extension-in-phase can be compensated by the commeasurably tiny quantity of density-in-phase, which reduces proportionally the effective time as to the system. Furthermore, action conservation can describe nonequilibrium states and processes unlike energy conservation implying equilibrium.

However Mehra states that "one might say that Gibbs succeeded very well in founding equilibrium thermodynamics on a statistical basis. But due to the great difficulties that existed at

Boltzmann was who associated it with the atomistic hypothesis borrowed from chemistry and thus "materialized" it. 
that time, and still do, he could not in the same way describe the gradual changes of the macroscopic properties in their approach to equilibrium." [50] This is only another way for one to say that Gibbs's theory describes both equilibrium and non-equilibrium states for the tool of phase space does not distinguish absolutely the two kinds of states to each other especially being quantized, i.e. "checked". However, this is an advantage in a sense, for anyone knows nowadays that the atoms are quantum entities rather than tiny material "balls" as in Boltzmann.

Only what one needs is to describe the time behavior of the thermodynamic systems in phase space:

"GIBBS' subtlety in achieving the maximum generality in his formulation must be stressed. He does not assume that thermodynamic systems are made up of molecules. He rather operates with abstract mechanical systems, and detects that thermodynamic systems obey the same laws as many particle mechanical systems, after a few reasonable approximations are made." [53]

Then Boltzmann's theory can be deduced as the particular case under the following two conditions:

$\checkmark$ The systems are to be described after a long enough time after changes (interactions), i.e. in equilibrium.

$\checkmark$ The phase space is not to be quantized.

$\checkmark$ Both conditions in fact mean one and the same: entropy (i.e. the number of microstates per a macrostate) to be big enough but correspondingly in Clausevius's and Boltzmann's definition.

Boltzmann's theory is not able to describe fast processes and states of small entropies, which are the informational ones and which turn out to be those in quantum mechanics. It forces Boltzmann's former "atoms" to be disjunctively separated in two big groups according to whether they obey the Pauli principle or not: either fermions or bosons.

E.T. Jaynes [54] emphasizes the "quantum essence" of entropy in a rather extraordinary way: "Even at the purely phenomenological level, entropy is an anthropomorphic concept. For it is a property, not of the physical system, but of the particular experiments you or I choose to perform on it." [52 $]^{10}$

Entropy just as information or quantum information is measured in units of elementary choices. However, "choice" is not an anthropomorphic concept, as there is no other way for any unordered and even unorderable state in nature to be ordered then by some series of choices.

Phase space can seem too parsimony. It allows to be deduced the First and Second Law but not the so-called Zeroth and Third Law of thermodynamics implying the absolute zero and prohibition of negative temperatures [55]. The collisions of atoms imply them in Boltzmann's theory. However they are spared and removed together with the atoms themselves in Gibbs's theory.

In fact, this is a part of the maximal generalization in it: It is able to incorporate not only the statistical thermodynamics of "atoms" entirely separated to each other, but furthermore the

${ }^{10}$ Also: "The difference is that energy is a property of the microstate, and so all observers, whatever macroscopic variables they may choose to define their thermodynamic states, must ascribe the same energy to a system in a given microstate. But they will ascribe different entropies to that microstate, because entropy is riot a property of the microstate, but rather of the reference class in which it is embedded." [54]

${ }^{11}$ The entropy of the velocities of a thermodynamic ensemble is what corresponds exactly to the degree of relative separation between the subsystems of a thermodynamic system. The absolute zero of temperature mixed entangled states in quantum thermodynamics, in which the elements are only partly separated to each other. Then the negative values of temperature originate from negative entropies and probabilities due to the mutual restriction of the degrees of freedom of entangled systems.

C. Tsallis introducing his fractal generalization of entropy noticed: "One of the most interesting is the fact that the inaccessible "temperatures" might belong to a finite interval that shrinks on the $T=0$ point in the $q \rightarrow 1$ limit" [56]. This means that the negative temperatures corresponds both to inseparable entangled states and over-separated fractal spaces (states). This is easily explainable for the inseparable entangled state in space of classical dimensionality $\mathrm{n}$ is equivalent to some over-separated fractal state in space of classical dimensionality $n+k(n, k=$ $1,2,3 \ldots$.$) . Or in other words, both Gibbs and Tsallis entropy are$ relevant both to fractal and to entangled states.

However C. Tsalis stressed [59] a main property of Gibbs's entropy, "extensivity or additivity" which his concept generalizes, in fact, just as the theory of entanglement needs. A few partly alternative comments are possible:

$\checkmark$ Tsallis's entropy corresponds more exactly to Gibbs's theory [57] than his own definition being a too literal generalization of Boltzmann's entropy.

$\checkmark$ Any entangled system considered to the subsystems can be interpreted as non-entangled to the whole of the system: that is in the Tsallis parameter $k \rightarrow 1$ limit, which corresponds to the state of equilibrium [61], [62]

$\checkmark$ The state of equilibrium is relative to the scale of time: "the binary property of the system being or not being in equilibrium is replaced by a continuous property of commonness" [63].

$\checkmark$ The interpretation of Gibbs's theory as implicitly meaning non-equilibrium states is incorrect for the condition of equilibrium was expressly stressed by him [53].

On the other hand, the sense of both negative and positive temperature as well is of the limit of absolute zero can be easily interpreted on that ground: The quantity of temperature ${ }^{11}$ measures the degree of relative separation between the subsystems of a thermodynamic system. Thus the relative mechanical motion of atoms or molecules in Boltzmann's theory should be understood as "anti-entanglement" just as entanglement in quantum information as "anti-motion". The absolute zero distinguishes the states of entanglement from those of mechanical motion.

The absolute zero is the boundary condition and borderline case, which Einstein [53] should discuss according to his declared intention, for the Newton mechanics applied to the "atoms" (the Boltzmann thermodynamics) to infer the Gibbs thermodynamics. Really, Einstein divided and emancipated to each other the two descriptions as the thermodynamics of the parts of a system (Boltzmann's theory) and the absolutely independent thermodynamics of the system as a whole (Gibbs's theory). Thus

corresponds to both zero velocities and zero entropy of a single microstate per a macrostate, i.e. to the entropy of a single and absolutely inseparable whole. Then the whole is the exact sum of immovable parts. The negative temperature means the whole to be less than the sum of parts, and the positive ones, to be more, therefore including empty space. The zero entropy of the velocities means both infinite (maximal) information of the velocities (or any quantity) and infinite entropy of the momenta (or the corresponding conjugate). 
his thermodynamics anticipated his later ideas and especially EPR as well as a future theory uniting the thermodynamics of entanglement, fractal dimensions, and classical statistic and mechanical ensembles.

In particular, this shows that Gibbs entropy is general enough. Its reasonable generalization seems to rather difficult ${ }^{12}$.

Phase space is just as (or more exactly, so) observable as atoms were in the age of Boltzmann and Gibbs. However, it unlike them has never claimed to be anything more than a theoretical instrument rather than reality.

There is a curious symmetry between the utilization of atoms and phase space correspondingly in Boltzmann's and Gibbs's theory: Both being unobservable allow of the system to be correspondingly described in terms of the parts or of the whole.

Then if Gibbs's theory is more general, the viewpoint of the whole turns out to be more general, too, and thus not perfectly symmetrical to that of the parts. They can be individualized barely after a long enough time, and before that they did not make sense or even not exist.

Einstein was who managed to restore the symmetry of whole and part in statistic thermodynamics in fact emancipating the descriptions in terms of the whole and the parts to each other.

Gibbs's theory can be called reductionist not less than that of Boltzmann only its reductionism is opposed: the atoms (elements) are reduced to properties or relations of the system by meditation of phase space.

Nowadays and post factum, Boltzmann's atomist reductionism seems to be justifiable and even prophetic as the atoms are incorporated in physical reality. However in the age of Boltzmann, Gibbs's elimination of the rather doubtful hypothesis about atoms was the more reasonable selection, moreover allowing of statistic thermodynamics to be formulated more generally.

This demonstrates once again that the reconstructing often depends on facts future to the selection for the very criterion of success suggests future events to determine ostensibly causally past theories.

One can summarize the active dimensions of reality in Gibbs's theory in comparison to Carnot's and Boltzmann's theories:

$\checkmark$ It is macroscopic as Carnot's and unlike Boltzmann's.

$\checkmark$ It constructs a rather sophisticated mathematical model as Boltzmann's and unlike Carnot's theory. Its relation to reality is neither so natural one as that of Carnot, nor so emancipated as Boltzmann's claiming to be that hidden reality, which grounds ours of empirical experience. Gibbs's theory involves theoretical and mathematical models only as tools for macroscopic thermodynamic reality to be investigated.

$\checkmark$ Gibbs's theory is holistic being skeptic to the existence of atoms without being anti-atomistic.

$\checkmark$ Thus it turns out to be non-relational and reductionist just as Boltzmann's is, eliminating however the elements, the "atoms" rather than the system as a whole. Both differs from Carnot's naïve harmonization of whole and parts in a cycle.

\section{EINSTEIN'S THEORY AND ITS CHANGE IN THE IMPLICIT CONCEPT OF REALITY}

The quite young and yet absolutely unknown Einstein wrote a few articles (1902-1904), the intention of which was statistical thermodynamics to be inferred absolutely rigorously from the laws of classical mechanics applied to big ensembles of atoms or molecules as well as to be suggested experiments, which might confirm or refuge their existence. "Einstein proceeded to derive the theorems of thermal equilibrium and the Second Law of Thermodynamics by the sole use of the mechanical equations and the theory of probability." [50]

His implicit concept of reality should be closer to that of Boltzmann rather than to that of Gibbs. Nevertheless he researched the construction of experiments for the atoms, which the pure theorist and philosopher Boltzmann did never. Unlike Boltzmann he did not attempt to reduce phenomenological thermodynamics to the mechanical movements of atoms and molecules, but did investigate the conditions, under which thermodynamics might be reduced to mechanics of atoms.

Thus, his implicit concept of reality is inherently and internally differential. It addressed the difference in the interpretation of reality in those two great theories of physics.

In fact this seems to be one of the secrets of his future successes and even ... seeming failures. Indeed:

$\checkmark$ Special relativity is situated between mechanics and the theory of electromagnetism. The implicit concept of reality in both is different. The action of mechanical forces is instantaneous at any distance, however limited to the constant light velocity in electromagnetism according to Michelson and Morley's experiments. Nevertheless he did not reduce mechanics to electromagnetism, but investigated the conditions, under which both could be consistent to each other.

$\checkmark$ General relativity is between special relativity and the theory of gravitation. The absolute and absolutely independent space and time, in which Newton's theory of gravity acts, contradict to the relative and unified space-time of special relativity. However, he did not attempt to reduce gravity to special relativity, but to harmonize both to each other.

$\checkmark$ His resistance to quantum mechanics can be located between it and his own theory of relativity both general and special. EPR demonstrated that quantum mechanics rests on a "spooky" action at a distance unlike both his theories of relativity. Thus in fact, the phenomena of entanglement were forecast though rejected in favour of the hypothesis of the alleged incompleteness of quantum mechanics. EPR rests a kind of atoms, the "elements of reality". However their reality was rejected in favour of the reality of quanta, in fact the cells of the "checked" phase space. Gibbs's theory reality wins against Boltzmann's.

$\checkmark$ His late research ran the space between electromagnetism and general relativity. It remained the empty the free option of a future non-quantum unified theory of gravity and electromagnetism.

The implicit methodology of Einstein demonstrates convincingly that the collision of realities of different physical theories is exceptionally fruitful. They suggest different viewpoints and answers what the world is but though interpretable

\footnotetext{
${ }^{12} \mathrm{~A}$ previous attempt [57], [58] seems to be even more unsuccessful.
} 
in a certain generalized and common theoretical "reference frame".

Differential realism investigates those a few especially important dimensions of reality, in which the "battle of realities" happens ...

So, Einstein's thermodynamics can be considered as the chronologically first application of his fundamental approach, which made him one of the greatest physicists of all time.

The essence of that "binocular" kind of models as to Einstein's thermodynamic can be explicit formulated by the assistance of two his later papers [64] and [65] commented in [60] about the quantity of thermodynamic probability (W) and Boltzmann's principle, i.e. the proportionality of entropy $(\mathrm{S})$ and $\log \mathrm{W}$ :

W implies some uncertainty, lack of knowledge about the macrostate (the one "ocular") in terms of the microstates (the other "ocular"), and thus "binocularly" reproduces the cognitive space of possible solutions, after which that space can be merely observed and the "events" in it described in "Gedankenexperiments".

One can say that quantum mechanics turns out to be a thermodynamic theory seen "binocularly" in that space. This originates from its fundamental principles formulated yet by Bohr: Unlike classical mechanics, it is a "binocular" or "dualistic" theory about both quantum entities and "apparatus" and thus about both microstates and macrostate implying a fundamental counterpart of W. This way for quantum mechanics to be seen can be called "thermodynamic mechanics" and opposed to Einstein's intention for some "mechanic thermodynamics" both however "dwelling" that space visible by Einstein's "pair of glasses".

Following Einstein's tradition of "Gedankenexperiments", let us begin shrink the "apparatus" more and more observing that space and its two "habitants": the "incomplete", but real quantum mechanics ("thermodynamic mechanics") and the alleged complete quantum mechanics ("mechanic thermodynamics"). The shrink of the apparatus causes some diminution of all microstates, and the microstates remain constant. This results into increasing $\mathrm{W}$ and decreasing $\mathrm{S}$.

When the size of the macrostates becomes commeasurable with that of the microstates, W begins to converge to 1 and $\mathrm{S}$ to 0 . This happens when the size of the apparatus has become commeasurable with that of the measured quantum entities.

When their sizes become equal to each other, $\mathrm{W}$ is just 1 , and $\mathrm{S}$ is 0 , and both habitants of that "binocular" space merge themselves into a single entity, which can be identified as classical mechanics. This merging is forced for the space is already so shrunk that there is room only for one habitant. The "totalitarian" principle is realized: "One microstate, one macrostate, one theory, probability one, but zero freedom (entropy)". Indeed classical mechanics is deterministic.

Some would stop here. Not we! The apparatus continues to shrink and its size is already less than that of the measured entities. The microstate is correspondingly bigger than that of the macrostate, and $\mathrm{W}>1$ : an extraordinary kind of probability, and $\mathrm{S}$ changes sign from plus to minus transforming iteslf into negative.

The case of probability bigger than 1 can be equivalently represented as that of negative probability if one considers the system of two independent events, the probability of the one of which is negative [68].

The negative probability implies the complex values of entropy: The room of the macrostate is already so tiny that a part of the microstate is already forced to go out in the space of the macrostate. Its probability is negative and its entropy is complex adding some purely imaginary entropy for the parts of the microstate remained outside of the macrostate. This is the world of quantum information and entanglement.

Let us exchange the inscriptions "MACROSTATE" and "MICROSTATE" to each other: Suddenly, we turn out to be in the starting point of the "Gedankenexpereiment", i.e. in our world. This is the quantum world if one exchanges the inscriptions "MACROSTATE" and "MICROSTATE". However one cannot even exchange them, but may look to the sky at night and to see the "microstates" as big as stars and nebulas ...

On the ground of that "Geadankenexperiment" one can reflect both Einstein's criticism to Boltzmann's principle and the essence of thermodynamic probability newly. The quantity of our "ignorance" $13, \mathrm{~W}^{*}=\mathbf{1}-\boldsymbol{W}$, about any physical quantity of any microstate makes physical sense in quantum mechanics as the thermodynamic probability $\mathrm{W}^{*}$ of the conjugate of the physical quantity at issue. The necessary condition is: $\log (1-W) \cong$ $\log 1-\log W=-\log W$, which is true only if $W \cong 0$, i.e. the "size" of the microstate is much, much less than that of the microstate: right the case in quantum mechanics. However, the above thought experiment demonstrates that quantum mechanics should be approximately valid and thus substitutable by a future (more) complete theory just as Einstein suggested if Boltzmann principle holds and the Boltzmann - Gibbs definition of entropy is relevant.

In fact the theorem about the absence of hidden variables [68], [69] demonstrate that quantum mechanics is complete and thus Boltzmann's principle and entropy should be only approximately valid right just to that limit of much, much bigger macrostates. Tsallis's entropy is one of the most relevant applicants to replace it [50]. Its parameter $\mathrm{k}$ can be always so adjusted to be satisfied the condition $\log W^{*} \cong \log W$ and even $\log W^{*}=\log W$.

Even more, under the conditions of the above thought experiments, an exact value of $\mathrm{k}$ corresponds to any size of the apparatus being shrunk: this value $\mathrm{k}$ can be inferred from a generalized Boltzmann principle where entropy $\mathrm{S}$ is substituted by Tsallis's entropy TS for the size of the shrunk apparatus (i.e. the "size" of a macrostate) is proportional to it under the condition of a standard and thus constant microstate.

The theorems about the absence of hidden variables in quantum mechanics [68], [67] can be interpreted as the absolute exact coincidence of its model and reality or as the inversion of the relation between the model and reality in comparison to classical physics. Here is how:

The model in quantum mechanics equates the degree of our ignorance about any physical quantity (i.e. the mismatch of the model to reality) to its conjugate, i.e. to another physical quantity and therefore to an "element of reality" thus transforming EPR's meaning of the term. After the difference between the model and reality is included in both model and reality, this implies formally their necessarily coincidence. The above adjustment of $\mathrm{k}$

\footnotetext{
${ }^{13} \mathrm{C}$. Tsallis also introduce his entropy as an (alternative) "measure of ignorance" discussing the non-universality of the Boltzmann - Gibbs (Shannon) entropy [67].
} 
according to the generalized Boltzmann principle follows that "ridiculous principle". Furthermore, the latter corresponds rather directly to the axiom of choice in mathematics.

Then one can postulate that "ridiculous principle": there is a special theory, right quantum mechanics, which is always and forever true, i.e. in any reality.

Nevertheless, Einstein's general relativity seems to be an apparent exclusion of the "ridiculous principle" at least until now if the curvature in a point in pseudo-Riemannian space does not corresponds somehow to the Tsallis parameter. And vice versa: if the ridiculous principle holds even to it, that correspondence seems to be necessary, linking entanglement and gravitation.

The common opinion is that Einstein's first attempt (19021904) is far not as successful as those in the next 1905 year. "Einstein's thermodynamical papers did not attract much attention." [50] One of the eventual causes might be that the unification of mechanics and thermodynamics implies at least some part of quantum mechanics:

Indeed the problem of black body radiation situated between electromagnetism and thermodynamics implied the fundamental Planck constant and thus concept of quantum leap. Furthermore, phase space checked by the Planck constant forces some non-zero difference between Boltzmann's and Gibbs's entropy. Einstein's approach implied also that kind of difference, but this might be only an accidental coincidence. Gibbs's theory could be interpreted as thermodynamics of system as a whole, and that of Boltzmann as thermodynamics of its parts. Then quantum mechanics seen as mechanics of the whole and parts might be the relevant bridge of unification.

Our problem to Einstein's thermodynamics does not include any estimation of its truth. Differential realism excludes that task in principle for it is tool to investigate only relation between two or more theories and their implicit concepts of reality rather than some alleged absolute relation of any theory to reality "by itself". This seems to be a metaphysical problem in a bad sense.

$\checkmark$ First of all, it is inherently and internally relative ${ }^{14}$ just as all Einstein's theories or ideas whether successful or unsuccessful. His implicit general methodology implies that relativity as this is demonstrated above and which distinguishes it from Carnot's, Boltzmann's, and Gibbs's theories. Carnot's does not suggests any problem about the whole and parts for they are naturally unified by the object of research, the steam-engine cycle. Boltzmann's and Gibbs's are more and less reductionist and thus non-relative.

$\checkmark$ This main property implies further for the concept of reality to be both macroscopic and microscopic as well as consisting both of a whole and of its parts. It generates a double or relative theoretical reality and model and thus outlines the possible space of unification. The double model is constructed intentionally to conserve the mismatch between the realities of the theories. Its main objectivity is to recreate the space of possible solution.

$\checkmark$ Carnot's, Boltzmann's, and Gibbs's models are constructed directly as solutions of their problem. Thus they more (Gibbs's and especially Boltzmann's) or less (Carnot's) dominate and ground reality. The double model of Einstein

\footnotetext{
${ }^{14}$ One can use the terms of relational and relationality for Einstein's of relative or relativity as referring to the meta-theory unlike Einstein's referring to the theory itself; but may not.
}

being intentionally partly inconsistent is not able to do this, and in fact this does not make much sense: Einstein himself called the type of his models "Gedankenexperiments". They are able to constitute self-developing or self-organizing theoretical reality, which Einstein as if only observed waiting for the solution of the problem from its standalone work by itself.

$\checkmark$ The same implies that the implicit concept of reality in his thermodynamics is both theoretical and observable.

\section{CONCLUSIONS \& FUTURE WORK}

The fundamental philosophical concept or category of reality turns out to be rather a fussy class sharing only "family resemblances" in the sense of Wittgenstein than the intersection of any common essence, which would allow for a general definition of "reality".

One can consider all the approaches of "selective realism' as "a box of tools" utilizable for investigating the implicit concept of reality in concrete scientific theories.

A few thermodynamic theories share a common implicit concept of reality as the empirically and experimentally accessible wholeness of the thermodynamic system distinguishing from each other by the different understand of its elements and thus, by the interpretation about their relation to the whole.

On the other hand the relation of "parts and whole" is a fundamental philosophical problem, which in the frames of the thermodynamic theories is supplied by an absolutely exact mathematical model as the corresponding conjecture underlying the theory rather than an only qualitative solution or preference as it is usual for philosophical doctrines.

The quantity of entropy, close to that of information, unifies the approaches of how one can describe mathematically bath parts and whole in the same terms and physical quantities for the thermodynamic description.

Thus, a fundamental philosophical and mathematical difference between the concept of reality in mechanics and thermodynamics can be outlined:

The mechanical elements of the whole are well-ordered in time as a certain trajectory. The thermodynamic elements, on the contrary, do no need a certain trajectory and admit a more general description invariant to the change between well-orderings of the same ensemble ${ }^{15}$.

The approach can serve as a heuristic tool to the implicit concept of reality in quantum mechanics after one has considered the coherent state before measurement "thermodynamically" (i.e. as an ensemble of all possible well-orderings (or "worlds") and the measured state "mechanically (i.e. bringing into the result a certain well-ordering due to the measuring itself rather than to quantum reality).

Furthermore, the transition from the "thermodynamic viewpoint before measurement" and the "mechanic viewpoint after measurement" can be interpreted as the exchange between two classes of frames or even, of reference frames "out of the (thermodynamic) system" and "within it" therefore hinting a possible generalization of the principle of relativity as an approach to the problem of quantum gravity.

\footnotetext{
${ }^{15}$ Just as a cardinal or ordinal number is invariant to the different well-
} orderings of the same set. 


\section{REFERENCES}

[1] E. Ruttkamp-Bloem. Re-enchanting Realism in Debate with Kyle Stanford. Journal for General Philosophy of Science, 44(1): 201-224 (2013).

[2] J. Saatsi and P. Vickers. Miraculous success? Inconsistency and untruth in Kirchhoff's diffraction theory. British journal for the philosophy of science, 62 (1) 29-46 (2011).

[3] G. Frost-Arnold. The no-miracles argument for realism: Inference to an unacceptable explanation. Philosophy of Science, 77(1): 35-58 (2010).

[4] U. Mäki. Scientific realism as a challenge to economics (and vice versa). Journal of Economic Methodology, 18(1): 1-12 (2011).

[5] T. Arabatzis. Conceptual Change and Scientific Realism: Facing Kuhn's Challenge. In: Re-framing the conceptual change approach in learning and instruction. S. Vosniadou, A. Baltas, X. Vamvakoussi (Eds.) Elsevier, in association with the European Association for Learning and Instruction, Amsterdam, London, Boston: 47-62 (2007).

[6] M. Colyvan. The ontological commitments of inconsistent theories. Philosophical Studies, 141(1): 115-123 (2008).

[7] E. McMullin. Comment: Selective anti-realism. Philosophical Studies: An International Journal for Philosophy in the Analytic Tradition, 61(1-2): 97-108 (1991).

[8] A. Fine. Piecemeal realism. Philosophical Studies: An International Journal for Philosophy in the Analytic Tradition, 61 (1-2): 79-96 (1991).

[9] P. Humphreys. Scientific Modeling - Computational Models. Philosophy of science, 69(3): S1-S11 (2002).

[10] P. K. Stanford. Scientific Realism, the Atomic Theory, and the CatchAll Hypothesis: Can We Test Fundamental Theories against All Serious Alternatives? The British Journal for the Philosophy of Science, 60, (2): 253-269 (2009).

[11] P. Vickers. Historical magic in old quantum theory? European journal for philosophy of science 2(1): 1-19 (2012).

[12] D. Harker. How to Split a Theory: Defending Selective Realism and Convergence without Proximity. The British Journal for the Philosophy of Science, 64(1): 79-106 (2013).

[13] D. Harker. Two arguments for scientific realism unified. Studies in History and Philosophy of Science Part A, 41(2): 192-202 (2010).

[14] M. Devitt. Rigid Application. Philosophical Studies: An International Journal for Philosophy in the Analytic Tradition, 125(2): 139-165 (2005).

[15] M. Devitt. Natural Kinds and Biological Realisms. In: Carving nature at its joints: natural kinds in metaphysics and science. J. K. Campbell, M. O'Rourke, M. H. Slater (Eds.) Cambridge, Mass.: MIT Press: 155173 (2011).

[16] J. Hricko. Jonathon Retail Realism and Wholesale Treatments of Theoretical Entitie [Preprint]. PhilSci-Archive (2014) http://philsciarchive.pitt.edu/id/eprint/10932 .

[17] D. Peters. What Elements of Successful Scientific Theories Are the Correct Targets for "Selective" Scientific Realism? Philosophy of Science, 81(3), 377-397 (2014).

[18] T. D. Lyons. Scientific Realism and the Stratagema de Divide et Impera. The British Journal for the Philosophy of Science, 57(3): 537560 (2006).

[19] P. K. Stanford. No Refuge for Realism: Selective Confirmation and the History of Science. Philosophy of science, 70(5): 913-925 (2003).

[20] S. Savitt. Selective Scientific Realism, Constructive Empiricism, and the Unification of Theories. Midwest Studies in Philosophy, 18(1): 154-165 (1993).

[21] K. Campbell. Selective realism in the philosophy of physics. The Monist, 77(1) ("The Ontology of Scientific Realism"): 27-46 (1994).

[22] B. H. Lavenda. A new perspective on thermodynamics. New York; London: Springer (2010).

[23] R. Fox. Watt's expansive principle in the work of Sadi Carnot and Nicolas Clément. Notes and records of the Royal Society of London, 24(2), 233-253 (1970).

[24] S. Carnot. Reflections on the motive power of heat. New York: John Wiley; London: Chapman \& Hall (1897).
[25] P. Lervig. On the structure of Carnot's theory of heat. Archive for History of Exact Sciences, 9(3): 222-239 (1972).

[26] G. Sarton. The Discovery of the Law of Conservation of Energy. Isis, 13(1): 18-44 (1929).

[27] M. K. Barnett. Sadi Carnot and the Second Law of Thermodynamics. Osiris, 13(1): 327-357 (1958).

[28] J. Srinivasan. Sadi Carnot and the second law of thermodynamics. Resonance, 6(11): 42-48 (2001).

[29] J. Sesták et al. Contribution by Lazare and Sadi Carnot to the caloric theory of heat and its inspirative role in thermodynamics. Journal of Thermal Analysis and Calorimetry, 97(2), 679-683 (2009).

[30] H. L. Callendar. The Caloric Theory of Heat and Carnot's Principle. Proceedings of the Physical Society of London, 23(1): 153-189 (1910).

[31] V. K. La Mer Some Current Misconceptions of N. L. Sadi Carnot's Memoir and Cycle. Science, 109 (2841): 598-598 (1949).

[32] J.N. Brønsted. The fundamental principles of energetics. The

London, Edinburgh, and Dublin Philosophical Magazine and Journal of Science, Series 7, 29(196) 449-470: (1940).

[33] D. S. Lemons and M. K. Penner. Sadi Carnot's contribution to the second law of thermodynamics. American Journal of Physics, 76(21): 21-25 (2008).

[34] S. A. Ulybin. Research, construction, calculations. S. Carnot's 200th birthday anniversary. Carnot heat theory. Thermodynamics. Phenomenological thermos mechanics. Chemical and Petroleum Engineering, 32(1): 1-8 (1996).

[35] E. Mendoza. The Life and Work of Sadi Carnot. The British Journal for the History of Science, 14(1): 75-78 (1981).

[36] S. Abe, S. Okuyama. Similarity between quantum mechanics and thermodynamics: entropy, temperature, and Carnot cycle. Physical review. E, Statistical, nonlinear, and soft matter physics, 83(2): 021121(3) (2011)

[37] C. Truesdell and S. Bharatha. The concepts and logic of classical thermodynamics as a theory of heat engines, rigorously constructed upon the foundation laid by S. Carnot and F. Reech. New York: Springer-Verlag (1977).

[38] E. Broda. Ludwig Boltzmann - Man, physicist, philosopher, biologist. Rheologica Acta, 21(4-5): 357-359 (1982).

[39] D. Flamm. Ludwig Boltzmann and his influence on science. Studies in the History and Philosophy of Science A, 14(4): 255-278 (1983).

[40] J. I. G. Tutor. Die Atomistik bei Ludwig Boltzmann. Zur wissenschaftlichen und philosophischen Bedeutung einer kontroversen Position am Ende des 19. Jahrhunderts. Journal for General Philosophy of Science, 35(2): 371-38 (2004).

[41] H. W. de Regt. Ludwig Boltzmann's Bildtheorie and scientific understanding. Synthese , 119(1-2): 113 - 134 (1999).

[42] F. Cardin and M. Favretti. On the Helmholtz-Boltzmann thermodynamics of mechanical systems. Continuum mechanics and thermodynamics, 16(1): 15-29 (2004).

[43] L. Boltzmann. Über die Beziehung eines allgemeine mechanischen Satzes zum zweiten Hauptsatze der Warmetheorie. In: Wissenschaftliche Abhandlungen (Herausgeber F. Hasenöhrl). Bd. II. Leipzig: Barth, 116-122 (1909).

[44] E.G.D. Cohen. Boltzmann and Einstein: Statistics and dynamics -

An unsolved problem. Pramana, 64(5): 635-643 (2005).

[45] S. G. Brush. Ludwig Boltzmann and the Foundations of Natural Science. In: I. M. Fasol-Boltzmann, G. L. (Hrsg.) Ludwig Boltzmann

(1844-1906). Zum hundertsten Todestag. Springer, Vienna, 65-80 (2006).

[48] Ludwig Boltzmann. On the necessity of atomic theories in physics. The Monist, 12(1): 65-79 (1901).

[47] W. Stiller. Ludwig Boltzmann - Pioneer of Atomistics and Evolution. Isotopenpraxis. Isotopes in Environmental and Health Studies, 22(8): 257-262 (1986).

[48] L. Boltzmann. Boltzmann's Philosophy Notes for Three Lectures (Fall 1903). Synthese. 119(1): 191 (1999).

[49] M. J. Klein. The Physics of J. Willard Gibbs in his Time. Physics Today, 43(9): 40-48 (1990).

[50] J. Mehra. Josiah Willard Gibbs and the Foundations of Statistical Mechanics. Foundations of Physics, 28(12): 1785-1815 (1998). 
[51] D. D. Nolte. The tangled tale of phase space. Physics Today, 63(4): 33-38 (2010).

[52] J. W. Gibbs. Elementary principles in statistical mechanics.

Scribner's sons, New York (1902).

[53] L. Navarro. Gibbs, Einstein and the Foundations of Statistical Mechanics. Archive for History of Exact Sciences, 53(2): 147-180 (1998).

[54] E T Jaynes. Gibbs vs Boltzmann entropies (1965) In: E.T. Jaynes: papers on probability, statistics, and statistical physics. Roger D Rosenkrantz ed. Dordrecht, Boston: D. Reidel; Hingham, MA. 7786 (1983).

[55] D. Frenkel and P. B. Warren. Gibbs, Boltzmann, and negative temperatures. American Journal of Physics, 83(2): 163-170 (2015).

[56] C. Tsallis. Possible Generalization of Boltzmann-Gibbs Statistics. Journal of Statistical Physics, 52(1-2): 479-487 (1988).

[57] W. Ochs. Basic properties of the generalized Boltzmann - Gibbs Shannon entropy. Reports of mathematical physics, 9(2): 135-155 (1976).

[58] W. Ochs. An unique characterization of the generalized Boltzmann Gibbs - Shannon entropy. Reports of mathematical physics, 9(3): 331354 (1976)

[59] C. Tsallis. Some comments on Boltzmann-Gibbs statistical mechanics. Chaos, Solitons \& Fractals, 6: 539-559 (1995).

[60] H. J. Haubold, A. M. Mathai, R. K. Saxena. Boltzmann-Gibbs Entropy Versus Tsallis Entropy: Recent Contributions to Resolving the Argument of Einstein Concerning "Neither Herr Boltzmann nor Herr Planck has Given a Definition of W"? Astrophysics and Space Science, 290(3-4): 241-245 (2004).

[61] C. Tsallis, D. Prato, and A. R. Plastino. Nonextensive Statistical Mechanics: Some Links with Astronomical Phenomena. Astrophysics and space science, 290(3-4): 259-274 (2004).

[62] N.G. de Almeida. Formal equivalence between Tsallis and extended Boltzmann-Gibbs statistics. Physica A: Statistical Mechanics and its Applications, 387(12): 2745-2749 (2008).

[63] D.A. Lavis. Boltzmann and Gibbs: An attempted reconciliation. Studies in History and Philosophy of Science Part B: Studies in History and Philosophy of Modern Physics, 36(2): 245-273 (2005).

[64] A. Einstein. Theorie der Opaleszenz von homogenen Flüssigkeiten und Flüssigkeitsgemischen in der Nähe des kritischen Zustandes [Annalen der Physik (Leipzig) 33: 1275-1298 (1910)]. In: The Collected Papers of Albert Einstein, The Swiss Years: Writings, 19091911. Volume 3, M.J. Klein, A.J. Kox, J. Renn, and Robert Schulmann (eds.). Princeton University Press, Princeton, 286-312 (1993).

[65] A. Einstein. Zum gegenwärtigen Stand des Strahlungsproblems [Physikalische Zeitschrift 10: 185-193 (1909)]. In: The Collected Papers of Albert Einstein, The Swiss Years: Writings, 1900-1909. Volume 2, J. Stachel (ed.), Princeton University Press, Princeton, 541553 (1993).

[66] V. Penchev. A Philosophical View on the Introduction of Negative and Complex Probability in Quantum Information. Philosophical Alternatives, 2012(1): 63-78.

[67] C. Tsallis. Remarks on the Nonuniversality of Boltzmann-Gibbs Statistical Mechanics. Fractals, 11(1): 319-326 (2003).

[68] J. von Neumann. Mathematische Grundlagen der Quantenmechanik, Berlin: Verlag von Julius Springer, 167-173 (1932).

[69] S. Kochen and E. Specker. The problem of hidden variables in quantum mechanics. Journal of Mathematics and Mechanics. 17 (1): 59-87 (1968) 